\title{
The Peripheral Hypotheses of Hypothalamic Aging
}

\author{
Zi-Jian Cai \\ CaiFortune Consulting, Suzhou, China \\ Email: hrsh8@126.com
}

How to cite this paper: Cai, Z.-J. (2018) The Peripheral Hypotheses of Hypothalamic Aging. Open Access Library Journal, 5: e4445.

https://doi.org/10.4236/oalib.1104445

Received: February 25, 2018

Accepted: March 23, 2018

Published: March 26, 2018

Copyright (๑) 2018 by author and Open Access Library Inc.

This work is licensed under the Creative Commons Attribution International License (CC BY 4.0).

http://creativecommons.org/licenses/by/4.0/

\section{(c) (7) Open Access}

\begin{abstract}
It is well known that the hypothalamic changes in control of hormones determine the chronological sequence of aging in mammals. For decades, it has been demonstrated in humans that the hypothalamic nuclei manifest heterogeneity in degeneration during aging, with the neuron number decreasing in both the suprachiasmatic nucleus (SCN) and the preoptic sexually dimorphic nucleus (SDN-POA) in the process of senescence, while the neuron number remains unchanged in the paraventricular nucleus (PVN). Recently, it was newly hypothesized some peripheral mechanisms responsible for the senescent changes of the hypothalamic nuclei. It was proposed by Cai that the decrease in slow-wave sleep (SWS) caused the degeneration of the suprachiasmatic nucleus (SCN). Besides, when reviewing the proposal by the European people in television about the senescent pathway for male reproduction on the degeneration of hypothalamic preoptic area by the common knowledge of reduction of sperm production from adipose accumulation in the middle/old age, it was as well demonstrated that the reduced testosterone level from the increased body fat caused the degeneration of the male preoptic sexually dimorphic nucleus (SDN-POA). It seems both the activity-dependent and hormonal regulation of the neuronal numbers are involved in the mechanisms causing the senescence of the hypothalamic nuclei. It is further pointed out that the paraventricular nucleus (PVN) maintaining its neuronal number unchanged in aging may cause many cellular and molecular changes of aging from chronic stress. It is expected that these preliminary considerations could elicit more investigations on the other peripheral causes for the hypothalamic aging, such as the cholesterol, hypertension, and so on.
\end{abstract}

\section{Subject Areas}

Developmental Biology, Geriatrics, Neuroscience, Zoology 


\section{Keywords}

Aging, Suprachiasmatic Nucleus, Slow Wave Sleep, Preoptic Sexually

Dimorphic Nucleus, Testosterone, Paraventricular Nucleus

\section{Introduction}

There have been many mechanisms postulated for the complex processes of senescence, including the oxidative accumulations in cells [1] [2] [3] [4], decrease in autophagic and proteolytic clearance within cells [5] [6] [7], telomere length-shortening [8] [9], chronological senescent changes in hypothalamic neuroendocrine [10] [11], thymic involution [12] [13], brain amyloid-beta accumulations [14] [15], and so on. Among these various senescent mechanisms, the hypothalamic neuroendocrine changes in the control of hormones [10] [11] determine the chronological sequence of aging in the ontogenetic life of vertebrates including mammals. In this article, it is attempted to review the recently proposed important hypotheses on the peripheral mechanisms causing the hypothalamic changes during aging, and by which it is hoped to elicit more comprehensive studies on the relevant investigations.

\section{Heterogeneity of Senescent Degeneration of Hypothalamic Nuclei}

\subsection{Heterogeneity in Degeneration of Hypothalamic Nuclei in Aging}

It has been demonstrated for decades of years that the hypothalamic nuclei manifest heterogeneity in degeneration during aging in mammals [10] [16] [17] [18] [19]. The paraventricular nucleus (PVN) is responsible for stress response and is functional throughout the lifespan, maintaining its neuron number in senescence in humans [10] [16]. In contrast, the suprachiasmatic nucleus (SCN), the main controller of circadian rhythm, is decreased in its number of neurons during the aging process in humans and marmosets [10] [16] [18]. Moreover, the sexually dimorphic nucleus in preoptic area (SDN-POA) declines sharply in cell number after aging in male humans and rats [10] [16] [17] [19]. In these respects, the hypothalamic nuclei manifest heterogeneity in degeneration during the complex processes of senescence.

\subsection{The Homogeneity of Amyloid-Beta in Brain in Contrast to the Heterogeneity of Hypothalamic Degeneration during Aging}

The brain aging is characterized as the accumulation of amyloid-beta [4] [14]. Brain aging is a plausible cause of senescence of hypothalamus. Recently, it was demonstrated that sleep helped biophysical clearance of amyloid-beta from the adult brain [15] [20], implicating that the neurons of brain were homogeneously subjected to the aging toxicity of amyloid-beta. In this regard, the various hypo- 
thalamic nuclei would in turn be subjected to the homogeneous toxicity of amyloid-beta from the forebrain, and would be homogeneously affected by amyloid-beta during aging.

However, as has just been mentioned, each cell group of the hypothalamic nuclei has its own specific pattern of aging, some decreasing while others maintaining in volume during aging [10] [16] [17] [18] [19]. Since the homogeneity of biophysical clearance of forebrain amyloid-beta [15] [20] would cause the homogeneous degeneration of the hypothalamic nuclei, the heterogeneity in degeneration of hypothalamic nuclei [10] [16] [17] [18] [19] indicates that the hypothalamic aging would result from the aging mechanisms other than the toxicity of amyloid-beta from forebrain.

\subsection{The Dissociation of the Forebrain Random Learning from the Patterns of Hypothalamic Aging}

Cai suggested early that, due to the neuronal plasticity of learning and memory, the gradual accumulation of various randomly learned memories in the limbic structures would inevitably imbalance and disorganize the emotional behaviors so that sleep should be developed in evolution to adjust and reorganize the emotions [21] [22] [23] [24]. Consequently, the influences from forebrain to the hypothalamus are random and various, changing from time to time. In this regard, if the hypothalamic aging resulted from the random and irregular plastic changes in the neural activities in forebrain, it would be difficult for the hypothalamus to specifically maintain the paraventricular nucleus (PVN) intact [10] [16] while only reduce the neurons in the suprachiasmatic nucleus (SCN) [10] [16] [18] and male preoptic sexually dimorphic nucleus (SDN-POA) [10] [16] [17] [19]. Therefore, it is as well deduced that the hypothalamic aging might result from the mechanisms other than those from the forebrain.

\section{The Hypothetic Peripheral Causes of Senescence of Hypothalamic Suprachiasmatic Nucleus (SCN)}

\subsection{The Hypothetic Aging Pathway from Skin to Hypothalamic Suprachiasmatic Nucleus (SCN) via Slow Wave Sleep}

Because of the above mentioned dissociation of the hypothalamic aging from the forebrain changes, Cai recently suggested a new peripheral hypothesis to explain the hypothalamic aging on suprachiasmatic nucleus (SCN) [25]. On the one hand, the continuous decrement in duration of slow-wave sleep (SWS) demonstrated in many senescent observations [25] [26] [27] [28] would cause the continuous functional degeneration of suprachiasmatic nucleus $(\mathrm{SCN})$ in hypothalamus during aging [10] [16] [18] [25]. On the other hand, the skin underwent aging from exposure to the environmental sunshine and oxygen as well as from genetic shortening of the telomere [25]. The skin senescence was reported repeatedly to result in reduction in the electrodermal activities [25], which would reduce the emotional responses and memories in brain, and 
would in turn reduce the requirement for slow wave sleep (SWS) [21] [22] [23] [25]. In these two respects, from skin senescence to slow wave sleep (SWS), it was formulated a new degenerative aging pathway to explain the chronological change of hypothalamic suprachiasmatic nucleus (SCN) during aging [25].

It is noted herein that the key to cause the aging of hypothalamic suprachiasmatic nucleus (SCN) [10] [16] [18] in this hypothesis was the decrement in duration of slow wave sleep (SWS) as demonstrated in many senescent observations [25] [26] [27] [28]. Besides the skin aging as the good cause to reduce the duration of slow wave sleep (SWS) in this hypothesis [25], there are still many other causes which may also reduce the duration of slow wave sleep (SWS), such as the cholesterol, hypertension, and so on. It is necessary to point out that the peripheral aging from the intracellular changes is homogenous within an organ, while that from cholesterol or angiemphraxis is relatively more heterogeneous, which requires more investigations.

\subsection{Further Evidence Supporting the Senescence of Hypothalamic Suprachiasmatic Nucleus (SCN) via the Slow Wave Sleep (SWS)}

The aging-related disorganization in expression of core clock genes in various brain regions supports this hypothetic pathway. It is common knowledge that the hippocampus is responsible for learning and memory [24]. It was demonstrated that the expression of Clock, Bmal1 and Per 2 genes in hippocampus lost circadian rhythm earlier than those in hypothalamic suprachiasmatic nucleus (SCN) [29] [30], implicating that the hippocampal circadian rhythm relevant to sleep and memories actually decreased earlier in response to the reduction of emotional responses, while such dysfunctions later began to affect the hypothalamic suprachiasmatic nucleus (SCN) [25]. If the degeneration of hypothalamic suprachiasmatic nucleus (SCN) during aging results from the changes in the secretion of growth hormone, there would not have shown this pattern of disorganization in expression of core clock genes earlier in the hippocampus and then in the hypothalamic suprachiasmatic nucleus (SCN) [29] [30].

Therefore, the decrement in duration of slow wave sleep (SWS) as demonstrated in many senescent observations [25] [26] [27] [28] is the most plausible cause for the degeneration of hypothalamic suprachiasmatic nucleus (SCN) during aging [10] [16] [18] [25]. In further, the senescent decrement in duration of slow wave sleep (SWS) [25] [26] [27] [28] may in turn result from the skin senescence as postulated by Cai [25], while may result also from other populationally more heterogeneously distributed causes such as the cholesterol, hypertension, and so on.

The hypothetic peripheral causes of senescence of hypothalamic suprachiasmatic nucleus (SCN) are shown in Figure 1. 
Skin senescence, manifesting as reduction in the electrodermal activities, results from: (1) exposure to the environmental sunshine; (2) exposure to the environmental oxygen; (3) genetic shortening of the telomere.

Slow wave sleep(SWS), for bodily restoration and emotional readjustment, is reduced in many senescent observations, because of the reduction of the emotional responses and memories in brain from the reduction in the electrodermal activities by skin senescence.

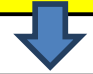

The reduction in slow wave sleep(SWS), demonstrated in many senescent observations, would cause the continuous functional degeneration of suprachiasmatic nucleus( $\mathrm{SCN})$ in hypothalamus controlling circadian rhythm during aging.

Figure 1. The peripheral causes of hypothalamic SCN aging.

\section{The Hypothetic Aging Pathway from Lipid to Preoptic Sexually Dimorphic Nucleus (SDN-POA) via Testosterone and Aromatase}

\subsection{The European View of Hypothalamic Aging of Male Preoptic Area (POA)}

In similarity to the aging pathway from skin to hypothalamic suprachiasmatic nucleus(SCN) via slow wave sleep (SWS), on July 4, 2016, the European people in television commented that there was an additional senescent pathway for male reproduction from the common knowledge of adipose accumulation and reduction of spermatogenesis in middle age/early old age to analogously elicit the degeneration of hypothalamic preoptic area (POA) [25] [31], while the senescence of female reproduction likewise resulting also from ovary menopause.

Because the senescent degeneration of hypothalamic suprachiasmatic nucleus (SCN) from skin senescence via slow wave sleep (SWS) would as well affect the reproductive system of animals, this pathway from adipose accumulation would provide an additional cause for the degeneration of male hypothalamic preoptic area (POA) [25] [31], making it degenerate sharply in the course of senescence as observed [10] [16] [17] [19].

\subsection{Regulation of Neuronal Numbers within the Male Preoptic Sexually Dimorphic Nucleus (SDN-POA) by Sex Hormones}

As mentioned above, it was indeed observed that the male sexually dimorphic nucleus in preoptic area (SDN-POA) declined in cell number after aging [10] [16] [17] [19]. Besides, it was even shown that the higher estradiol resulted in the neuronal loss in the preoptic sexually dimorphic nucleus (SDN-POA) in male rats [17], while the testosterone reversed the neuronal degeneration within the preoptic sexually dimorphic nucleus (SDN-POA) in male rats [19], demonstrating that the hormonal levels directly able to result in the neuronal degeneration within the male preoptic sexually dimorphic nucleus (SDN-POA).

In this regard, via looking at those affecting the hormonal levels of testosterone and estradiol [17] [19], it would be possible to find out the peripheral causes resulting in the neuronal degeneration within the preoptic sexually dimorphic 
nucleus (SDN-POA) during male aging.

\subsection{The Lipid Regulation of Enzyme Aromatase Converting Testosterone to Estradiol during Aging}

The European view on hypothalamic aging of preoptic area (POA) from adipose accumulation [25] [31] implicated the premise that it resulted from the intrinsic aging process genetically set in development and growth. The morphogenetic development in animals is controlled by both induction and termination [32]. Induction initiates the morphogenetic development while termination controls the final shape of the generated organ during morphogenesis [32]. Many animal models can demonstrate the induction and termination as the key developmental control for the morphological changes, such as the sexual dimorphism in mammary glands, the epidermal scale in reptiles, the tail metamorphosis in amphibians, the variation in limb digits in vertebrates, and so on [32].

In vertebrates, the fat depot sizes reach a peak by middle age or early old age, followed by a substantial decline in advanced old age [33]. Fat tissue growth occurs by increase in size and number of fat cells. Beginning at middle age, the subcutaneous fat loses earlier and consequently the intra-abdominal fat increases relatively in the course toward aging [33], resulting in insulin resistance and increased risk of atherosclerosis and diabetes, even in lean subjects [33]. Nutrients, hormonal effectors such as insulin, leptin, IGF-1, glucocorticoids, and so on are responsible for the regulation of adipose size and redistribution in development and aging [33] [34].

The enzyme aromatase in vertebrates converts the testosterone to estradiol and decreases the testosterone levels [35] [36]. The aromatase is expressed in the differentiated adipose cells [35]. Accordingly, Cohen suggested earlier that the increase in adipose tissue in middle age be associated with an increase in the enzyme aromatase and lead to diminished testosterone levels during male aging [36].

It seems not convincing to overemphasize the special role of only one enzyme as aromatase in disturbing the male reproductive functions in aging. However, aromatase is really a remarkable enzyme and even plays more dramatic roles in sex determination. Aromatase is an evolutionary conserved enzyme present in amphioxus [37], fishes [38] [39], amphibians [40], reptiles [41], and mammals including humans [35] [36]. It is suggested as the key enzyme in the epigenetic determination of sex as male or female during ontogeny in many species of vertebrates [42], as demonstrated in African cichlid fishes [39], Japanese frog Ranarugosa [40] and some reptiles [41].

In this regard, due to the significant effects of aromatase demonstrated in sex determination in many vertebrates [39] [40] [41] [42], it is obvious that the increase in aromatase from increment of fat size in middle age is sufficient to significantly disturb the male reproductive system via converting the testosterone to estradiol. 


\subsection{The Disturbance on Male Sexual Hormones and Hypothalamus from Adipose and Aromatase in Aging}

The disturbance on male reproductive system from adipose and aromatase in aging can even be assayed in vivo. In one report, it was demonstrated that the free and bioavailable estradiol levels decreased modestly with age as did the ratio of free testosterone to free estradiol, the latter testifying to the age-related increase in aromatization of testosterone [43]. The estradiol levels were highly, significantly and positively related to the body fat mass [43]. In another paper comparing BMI in relation to sperm count, it was shown that the overweight and obesity were associated with an increased prevalence of azoospermia or oligozoospermia [44].

In this regard, due to the regulation of neuronal numbers within the preoptic sexually dimorphic nucleus (SDN-POA) by the testosterone and estradiol [17] [19], the increment of adipose and aromatase converting the testosterone to estradiol in aging [43] would cause the neuronal degeneration within the male sexually dimorphic nucleus in preoptic area (SDN-POA).

Because the degeneration of suprachiasmatic nucleus (SCN) from skin aging via slow wave sleep (SWS) would also affect the function of reproduction, the cause from adipose accumulation for the degeneration of male preoptic sexually dimorphic nucleus (SDN-POA) would make an additional contribution to its senescent degeneration, so that make its cells decline sharply after aging as observed [10] [16] [17] [19].

\subsection{The Association of Testosterone with Hypertension during Aging}

It is noted that the regulation of the testosterone by lipid may not solely via the rise in aromatase during male aging, even though the rise in aromatase is sufficient to remarkably disturb the male reproductive system in senescence. In parallel, it has also been demonstrated that the level of testosterone is associated with the risk of hypertension [45] [46] [47]. Due to the limited data available, it is uncertain about the regulation of testosterone by hypertension during aging. It is necessary to carry out more investigations on this issue.

\subsection{Section Summary}

In this section, it is demonstrated the European view on the aging of male hypothalamic preoptic area (POA) from adipose accumulation and the relevant supporting facts, as the followings: 1) The genetic increase and redistribution of fat tissue in male middle age. 2) The aromatase, able to determine the sex differentiation in vertebrates, being expressed in adipose tissue to convert the testosterone to estradiol during male aging. 3) The observed disturbance on male reproductive hormones from increment of adipose and aromatase in aging. 4) In response to the hormonal outcomes from the rise in aromatase, the neuronal degeneration within the sexually dimorphic nucleus in preoptic area (SDN-POA) 
after male aging. 5) The necessity to carry out more investigations about the regulation of testosterone by hypertension during aging.

It is necessary to point out that, for the adipose cause of neuronal degeneration within the sexually dimorphic nucleus in preoptic area (SDN-POA) after male aging, the European view in television adopted the reduction of spermatogenesis as the key mediator, while in literature there were evidences that the ratio of testosterone to estradiol played the key role.

The hypothetic peripheral causes of senescence of male hypothalamic sexually dimorphic nucleus in preoptic area (SDN-POA) are shown in Figure 2.

\section{The Intact Hypothalamic Paraventricular Nucleus (PVN) during Aging}

The hypothalamic paraventricular nucleus (PVN), responsible for stress response, is functional throughout the lifespan and maintains its neuron number in senescence in humans [10] [16]. Obviously, the stress response is thus maintained in aging, which may cause decrease in proteolytic clearance within peripheral cells during aging [7]. Besides, it was recently reviewed that the psychological stress might lead to aging via many other molecular processes in cells, such as the change in length of telomere, increase in DNA damage, alternation in immunological inflammation, and so on [48].

In contrast to the senescent decrease in number of neurons in hypothalamic suprachiasmatic nucleus (SCN) [10] [16] [18] and male preoptic sexually dimorphic nucleus (SDN-POA) [10] [16] [17] [19] in humans, the preservation of the neurons and functions of PVN [10] [16] in further indicates that: 1) The hypothalamic aging would result from the aging mechanisms other than the toxicity of amyloid-beta from forebrain, since the homogeneity of biophysical clearance of forebrain amyloid-beta [15] [20] would cause the homogeneous degeneration of the hypothalamic nuclei. 2) The hypothalamic aging would dissociate from the aging mechanisms relevant to the random forebrain neuronal plasticity due to learning and memory [21] [22] [23] [24], since the random and irregular changes in the neural activities in forebrain from learning make it difficult for the hypothalamus to maintain the PVN intact [10] [16] while only reduce the

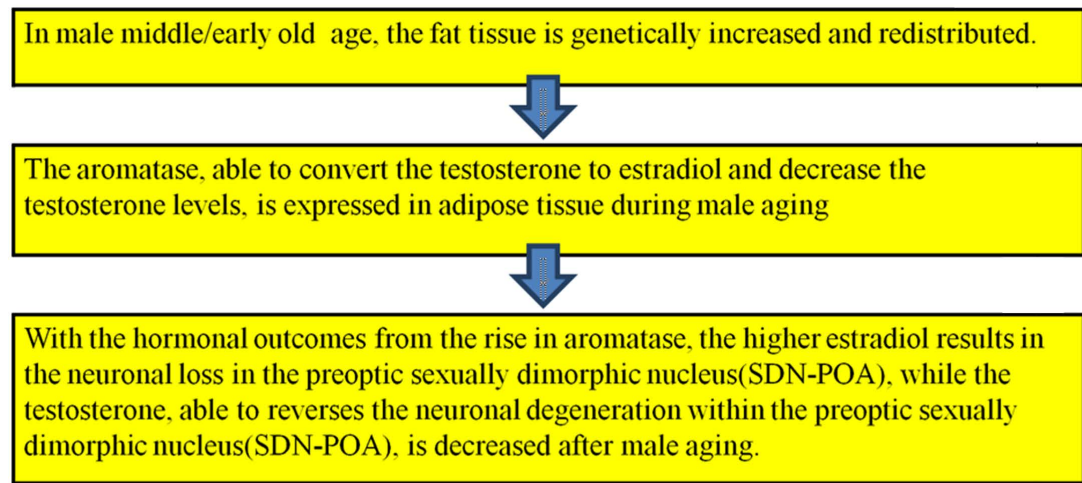

Figure 2. The peripheral causes of male hypothalamic SDN-POA aging. 
neurons in the suprachiasmatic nucleus (SCN) [10] [16] [18] and male preoptic sexually dimorphic nucleus (SDN-POA) [10] [16] [17] [19].

\section{Discussions}

The hypothalamic nuclei manifest heterogeneity in degeneration during aging in mammals [10] [16] [17] [18] [19]. The suprachiasmatic nucleus (SCN) is decreased in its number of neurons during the aging process in humans and marmosets [10] [16] [18], while the male sexually dimorphic nucleus in preoptic area (SDN-POA) declines sharply in cell number after aging in humans and rats [10] [16] [17] [19]. However, the paraventricular nucleus (PVN) maintains its neuronal number unchanged during the aging in humans [10] [16].

The plausible peripheral mechanisms for causing the heterogeneity in degeneration of hypothalamic nuclei in aging are reviewed in this article, especially some newly hypothesized mechanisms.

For the suprachiasmatic nucleus (SCN), Cai suggested [25] that the continuous decrement in duration of slow-wave sleep (SWS) in many senescent observations [25] [26] [27] [28] would cause the continuous functional degeneration of suprachiasmatic nucleus (SCN) in hypothalamus during aging [10] [16] [18] [25]. The observations that the hippocampal circadian rhythm decreased earlier than the hypothalamic suprachiasmatic nucleus (SCN) [29] [30] supported this explanation [25]. Many peripheral causes can reduce the duration of SWS during aging, including the skin aging proposed by Cai [25], and such uncertain factors as the cholesterol, hypertension, and so on, which requires more investigations.

For the preoptic sexually dimorphic nucleus (SDN-POA), the European people in television suggested [25] [31] that the increased body fat would cause the degeneration of this hypothalamic nucleus during male aging. Many peripheral pathways may mediate this mechanism, especially the testosterone and estradiol levels which have been demonstrated to be able to regulate the neuronal numbers in this hypothalamic nucleus [17] [19]. The rise in aromatase can convert the testosterone to estradiol during aging [35] [36] [43]. Besides, it is necessary to carry out more investigations on the regulation of testosterone by hypertension during aging.

For the paraventricular nucleus (PVN) responsible for stress response, it maintains its neuronal number unchanged during aging [10] [16] in contrast to the decrease in both the suprachiasmatic nucleus (SCN) [10] [16] [18] and the male sexually dimorphic nucleus in preoptic area (SDN-POA) [10] [16] [17] [19] in humans. In this regard, it can demonstrate many peripheral changes of aging from chronic stress, including the decrease in proteolytic clearance within peripheral cells [7], change in length of telomere [48], increase in DNA damage [48], alternation in immunological inflammation [48], and so on.

Because the degeneration of suprachiasmatic nucleus (SCN) from reduction in slow wave sleep (SWS) would also affect the function of male reproductive 
system, the adipose accumulation causing degeneration of the male preoptic sexually dimorphic nucleus (SDN-POA) would make an additional contribution to its degeneration [25] [31], making its cells decline sharply after aging as observed [10] [16] [17] [19].

It is interesting to inspect the evolutionary differences of these two degenerative mechanisms addressing to the two hypothalamic nuclei during aging. In phylogeny, the forebrain slow wave in sleep is only present in vertebrates more advanced than reptiles [21] [22], so that the senescent pathway from the slow wave sleep (SWS) to degeneration of suprachiasmatic nucleus (SCN) [25] would be applicable only to the higher vertebrates. Whereas, because aromatase is present in all vertebrates including the fishes, amphibians, reptiles and mammals [35]-[41], the aging pathway from adipose accumulation to degeneration of male hypothalamic preoptic sexually dimorphic nucleus (SDN-POA) [25] [31] would be applicable to all vertebrates, more ancient and conserved in evolution than the degeneration of suprachiasmatic nucleus (SCN) from slow wave sleep (SWS).

It is further noted that, as the hypothesized degeneration of suprachiasmatic nucleus ( $\mathrm{SCN}$ ) during aging results from the continuous decrement in duration of slow-wave sleep (SWS) [25], it belongs to the functionally usage-dependent or activity-dependent regulation of neuronal numbers in the neuronal pathways. Whereas, as the hypothesized degeneration of male preoptic sexually dimorphic nucleus (SDN-POA) during aging results from the hormonal regulation of testosterone and estradiol on the neuronal numbers of this hypothalamic nucleus [25] [31], it belongs to the hormonal regulation of neuronal numbers. Therefore, both the activity-dependent and hormonal regulation of the neuronal numbers are adopted for the senescent changes of these hypothalamic nuclei during aging.

\section{Perspectives}

These two new theories of the peripheral aging pathways, from reduction in slow wave sleep (SWS) to degeneration of hypothalamic suprachiasmatic nucleus (SCN) and from rise of adipose/aromatase to degeneration of hypothalamic preoptic sexually dimorphic nucleus (SDN-POA) via testosterone, are significant. On the one hand, they provide the explanations to account for the chronological ontogenetic changes of hypothalamic suprachiasmatic nucleus (SCN) and male preoptic sexually dimorphic nucleus (SDN-POA) during aging. On the other hand, they provide the useful theories to guide the therapeutic efforts against aging in future. With regard to the degeneration of suprachiasmatic nucleus (SCN) during aging, the therapeutic anti-aging efforts should be more devoted to skin protection, sleep recovery and so on. With regard to the degeneration of male preoptic sexually dimorphic nucleus (SDN-POA) during aging, the therapeutic anti-aging efforts should be more devoted to control of energy intake, exercise to reduce adipose and so on. With regard to the intactness of 
paraventricular nucleus (PVN), the therapeutic anti-aging efforts should be more devoted to decrease of oxidative and psychological stress, and so on. In these regards, these new theories would be important to biomedical sciences.

\section{Conclusion}

In this article, the peripheral mechanisms are addressed resulting in the heterogeneity in degeneration of hypothalamic nuclei during aging in mammals. The neuron numbers decrease in both the suprachiasmatic nucleus (SCN) and the male preoptic sexually dimorphic nucleus (SDN-POA) during the process of aging, while the neuron number remains unchanged in the paraventricular nucleus (PVN). The decrease in slow wave sleep (SWS) causes the degeneration of the suprachiasmatic nucleus (SCN), while the reduced testosterone level from the increased body fat causes the degeneration of the male preoptic sexually dimorphic nucleus (SDN-POA). It seems both the activity-dependent and hormonal regulation of the neuronal numbers are adopted in the senescent changes of these hypothalamic nuclei. The paraventricular nucleus (PVN) responsible for stress response maintains its neuronal number unchanged during the aging in humans, causing many peripheral cellular and molecular changes of aging from chronic stress, including the decrease in proteolytic clearance, change in length of telomere, increase in DNA damage, alternation in immunological inflammation, and so on. These preliminary considerations are expected to elicit more comprehensive investigations on the peripheral causes of the heterogeneity in degeneration of the hypothalamic nuclei during aging, extending to consider other populationally more heterogeneously distributed causes such as the cholesterol, hypertension, and so on.

\section{Acknowledgements}

It is herein acknowledged that Mingxun Cai paid the Open Access publication fee of this paper.

\section{Conflict of Interest}

The author declares no conflict of interest nor financial support for this work.

\section{References}

[1] Kim, S.K. (2007) Common Aging Pathways in Worms, Flies, Mice and Humans. Journal of Experimental Biology, 210, 1607-1612. https://doi.org/10.1242/jeb.004887

[2] Zahn, J.M. and Kim, S.K. (2007) Systems Biology of Aging in Four Species. Current Opinion in Biotechnology, 18, 355-359.

https://doi.org/10.1016/j.copbio.2007.07.004

[3] Ishii, N., Ishii, T. and Hartman, P.S. (2007) The Role of the Electron Transport SDHC Gene on Lifespan and Cancer. Mitochondrion, 7, 24-28. https://doi.org/10.1016/j.mito.2006.11.012

[4] Sompol, P., Ittarat, W., Tangpong, J., Chen, Y., Doubinskaia, I., Batinic-Haberle, I., 
Abdul, H.M., Butterfield, D.A. and St Clair, D.K. (2008) A Neuronal Model of Alzheimer's Disease: An Insight into the Mechanisms of Oxidative Stress-Mediated Mitochondrial Injury. Neuroscience, 153, 120-130.

https://doi.org/10.1016/j.neuroscience.2008.01.044

[5] Nakamura, S. and Yoshimori, T. (2018) Autophagy and Longevity. Molecules and Cells, 41, 65-72.

[6] Hubbard, V.M., Valdor, R., Macian, F. and Cuervo, A.M. (2012) Selective Autophagy in the Maintenance of Cellular Homeostasis in Aging Organisms. Biogerontology, 13, 21-35. https://doi.org/10.1007/s10522-011-9331-x

[7] Koga, H., Kaushik, S. and Cuervo, A.M. (2011) Protein Homeostasis and Aging: The Importance of Exquisite Quality Control. Ageing Research Reviews, 10, 205-215. https://doi.org/10.1016/j.arr.2010.02.001

[8] Aubert, G. and Lansdorp, P.M. (2008) Telomeres and Aging. Physiological Reviews, 88, 557-579. https://doi.org/10.1152/physrev.00026.2007

[9] Blasco, M.A. (2007) Telomere Length, Stem Cells and Aging. Nature Chemical Biology, 3, 640-649. https://doi.org/10.1038/nchembio.2007.38

[10] Hofman, M.A. (1997) Lifespan Changes in the Human Hypothalamus. Experimental Gerontology, 32, 559-575. https://doi.org/10.1016/S0531-5565(96)00162-3

[11] Aguilera, G. (2011) HPA Axis Responsiveness to Stress: Implications for Healthy Aging. Experimental Gerontology, 46, 90-95.

https://doi.org/10.1016/j.exger.2010.08.023

[12] Bar-Dayan, Y., Afek, A., Bar-Dayan, Y., Goldberg, I. and Kopolovic, J. (1999) Proliferation, Apoptosis and Thymic Involution. Tissue and Cell, 31, 391-396.

https://doi.org/10.1054/tice.1999.0001

[13] Taub, D.D. and Longo, D.L. (2005) Insights into Thymic Aging and Regeneration. Immunological Reviews, 205, 72-93. https://doi.org/10.1111/j.0105-2896.2005.00275.x

[14] Wang, X.L., Su, B., Perry, G., Smith, M.A. and Zhu, X.W. (2007) Insights into Amyloid- $\beta$ Induced Mitochondrial Dysfunction in Alzheimer Disease. Free Radical Biology and Medicine, 43, 1569-1573. https://doi.org/10.1016/j.freeradbiomed.2007.09.007

[15] Kang, J.E., Lim, M.M., Bateman, R.J., Lee, J.J., Smyth, L.P., Cirrito, J.R., Fujiki, N., Nishino, S. and Holtzman, D.M. (2009) Amyloid-Beta Dynamics Are Regulated by Orexin and the Sleep-Wake Cycle. Science, 326, 1005-1007. https://doi.org/10.1126/science.1180962

[16] Zhou, J.-N. and Swaab, D.F. (1999) Activation and Degeneration during Aging: A Morphometric Study of the Human Hypothalamus. Microscopy Research and Technique, 44, 36-48. https://doi.org/10.1002/(SICI)1097-0029(19990101)44:1<36::AID-JEMT5>3.0.CO;2$\underline{\mathrm{F}}$

[17] Hsieh, Y.L., Hsu, C., Yang, S.L., Hsu, H.K. and Peng, M.T. (1996) Estradiol Modulation of Neuron Loss in the Medial Division of Medial Preoptic Nucleus in Rats during Aging. Gerontology, 42, 18-24. https://doi.org/10.1159/000213766

[18] Engelberth, R.C., Silva, K.D., Azevedo, C.V., Gavioli, E.C., dos Santos, J.R., Soares, J.G., Nascimento Jr., E.S., Cavalcante, J.C., Costa, M.S. and Cavalcante, J.S. (2014) Morphological Changes in the Suprachiasmatic Nucleus of Aging Female Marmosets (Callithrix jacchus). BioMed Research International, 2014, Article ID: 243825.

[19] Panzica, G.C., García-Ojeda, E., Viglietti-Panzica, C., Thompson, N.E. and Ottin- 
ger, M.A. (1996) Testosterone Effects on Vasotocinergic Innervation of Sexually Dimorphic Medial Preoptic Nucleus and Lateral Septum during Aging in Male Quail. Brain Research, 712, 190-198. https://doi.org/10.1016/0006-8993(95)01386-5

[20] Xie, L., Kang, H., Xu, Q., Chen, M.J., Liao, Y., Thiyagarajan, M., O’Donnell, J., Christensen, D.J., Nicholson, C., Iliff, J.J., Takano, T., Deane, R. and Nedergaard, M. (2013) Sleep Drives Metabolite Clearance from the Adult Brain. Science, 342, 373-377. https://doi.org/10.1126/science.1241224

[21] Cai, Z.-J. (1991) The Functions of Sleep: Further Analysis. Physiology \& Behavior, 50, 53-60. https://doi.org/10.1016/0031-9384(91)90497-C

[22] Cai, Z.-J. (1995) An Integrative Analysis to Sleep Functions. Behavioural Brain Research, 69, 187-194. https://doi.org/10.1016/0166-4328(95)00005-E

[23] Cai, Z.-J. (2016) Progressions of Sleep, Memory and Depression Applicable to Psychoanalysis: A Review. Current Psychiatry Reviews, 12, 240-245. https://doi.org/10.2174/1573400512666160610083505

[24] Cai, Z.-J. (1990) The Neural Mechanism of Declarative Memory Consolidation and Retrieval: A Hypothesis. Neuroscience \& Biobehavioral Reviews, 14, 295-304. https://doi.org/10.1016/S0149-7634(05)80039-9

[25] Cai, Z.-J. (2016) A Hypothetic Aging Pathway from Skin to Hypothalamic Suprachiasmatic Nucleus via Slow Wave Sleep. Sleep Science, 9, 212-215. https://doi.org/10.1016/j.slsci.2016.09.004

[26] Ehlers, C.L. and Kupfer, D.J. (1989) Effects of Age on Delta and REM Sleep Parameters. Electroencephalography and Clinical Neurophysiology, 72, 118-125. https://doi.org/10.1016/0013-4694(89)90172-7

[27] Espiritu, J.R. (2008) Aging-Related Sleep Changes. Clinics in Geriatric Medicine, 24, 1-14. https://doi.org/10.1016/j.cger.2007.08.007

[28] Hirshkowitz, M., Moore, C.A., Hamilton3rd, C.R., Rando, K.C. and Karacan, I. (1992) Polysomnography of Adults and Elderly: Sleep Architecture, Respiration, and Leg Movement. Journal of Clinical Neurophysiology, 9, 56-62. https://doi.org/10.1097/00004691-199201000-00006

[29] Duncan, M.J., Prochot, J.R., Cook, D.H., Tyler Smith, J. and Franklin, K.M. (2013) Influence of Aging on Bmall and Per2 Expression in Extra-SCN Oscillators in Hamster Brain. Brain Research, 1491, 44-53. https://doi.org/10.1016/j.brainres.2012.11.008

[30] Wyse, C.A. and Coogan, A.N. (2010) Impact of Aging on Diurnal Expression Patterns of CLOCK and BMAL1 in the Mouse Brain. Brain Research, 1337, 21-31. https://doi.org/10.1016/j.brainres.2010.03.113

[31] Cai, Z.-J. (2017) The Adipose Cause of Senescence of Male Hypothalamic Preoptic Nucleus: An European View. Research \& Reviews: Neuroscience, 1, 21-26.

[32] Cai, Z.-J. (2015) Termination of Organogenesis as Intrinsic Constraint on Animal Development and Evolution: A Theory. Open Access Library Journal, 2, e1646. https://doi.org/10.4236/oalib.1101646

[33] Cartwright, M.J., Tchkonia, T. and Kirkland, J.L. (2007) Aging in Adipocytes: Potential Impact of Inherent, Depot-Specific Mechanisms. Experimental Gerontology, 42, 463-471. https://doi.org/10.1016/j.exger.2007.03.003

[34] Carrascosa, J.M., Ros, M., Andrés, A., Fernández-Agulló, T. and Arribas, C. (2009) Changes in the Neuroendocrine Control of Energy Homeostasis by Adiposity Signals during Aging. Experimental Gerontology, 44, 20-25.

https://doi.org/10.1016/j.exger.2008.05.005 
[35] Yamada, K. and Harada, N. (1990) Expression of Estrogen Synthetase (P-450 Aromatase) during Adipose Differentiation of 3T3-L1 Cells. Biochemical and Biophysical Research Communications, 169, 531-536. https://doi.org/10.1016/0006-291X(90)90363-R

[36] Cohen, P.G. (2001) Aromatase, Adiposity, Aging and Disease. The Hypogonadal-Metabolic-Atherogenic-Disease and Aging Connection. Medical Hypotheses, 56, 702-708. https://doi.org/10.1054/mehy.2000.1169

[37] Callard, G.V., Tarrant, A.M., Novillo, A., Yacci, P., Ciaccia, L., Vajda, S., Chuang, G.Y., Kozakov, D., Greytak, S.R., Sawyer, S., Hoover, C. and Cotter, K.A. (2011) Evolutionary Origins of the Estrogen Signaling System: Insights from Amphioxus. The Journal of Steroid Biochemistry and Molecular Biology, 127, 176-188. https://doi.org/10.1016/j.jsbmb.2011.03.022

[38] Zhang, Y., Zhang, S., Lu, H., Zhang, L. and Zhang, W. (2014) Genes Encoding Aromatases in Teleosts: Evolution and Expression Regulation. General and Comparative Endocrinology, 205, 151-158. https://doi.org/10.1016/j.ygcen.2014.05.008

[39] Böhne, A., Heule, C., Boileau, N. and Salzburger, W. (2013) Expression and Sequence Evolution of Aromatase cyp19al and Other Sexual Development Genes in East African Cichlid Fishes. Molecular Biology and Evolution, 30, 2268-2285. https://doi.org/10.1093/molbev/mst124

[40] Nakamura, M. (2013) Is a Sex-Determining Gene(s) Necessary for Sex-Determination in Amphibians? Steroid Hormones May Be the Key Factor. Sexual Development, 7, 104-114. https://doi.org/10.1159/000339661

[41] Girondot, M., Ben Hassine, S., Sellos, C., Godfrey, M. and Guillon, J.M. (2010) Modeling Thermal Influence on Animal Growth and Sex Determination in Reptiles: Being Closer to the Target Gives New Views. Sexual Development, 4, 29-38. https://doi.org/10.1159/000280585

[42] Nakamura, M. (2010) The Mechanism of Sex Determination in Vertebrates-Are Sex Steroids the Key-Factor? Journal of Experimental Zoology Part A: Ecological Genetics and Physiology, 313A, 381-398. https://doi.org/10.1002/jez.616

[43] Vermeulen, A., Kaufman, J.M., Goemaere, S. and van Pottelberg, I. (2002) Estradiol in Elderly Men. The Aging Male, 5, 98-102. https://doi.org/10.1080/tam.5.2.98.102

[44] Sermondade, N., Faure, C., Fezeu, L., Shayeb, A.G., Bonde, J.P., Jensen, T.K., Van Wely, M., Cao, J., Martini, A.C., Eskandar, M., Chavarro, J.E., Koloszar, S., Twigt, J.M., Ramlau-Hansen, C.H., Borges Jr., E., Lotti, F., Steegers-Theunissen, R.P., Zorn, B., Polotsky, A.J., La Vignera, S., Eskenazi, B., Tremellen, K., Magnusdottir, E.V., Fejes, I., Hercberg, S., Lévy, R. and Czernichow, S. (2013) BMI in Relation to Sperm Count: An Updated Systematic Review and Collaborative Meta-Analysis. Human Reproduction Update, 19, 221-231.

https://doi.org/10.1093/humupd/dms050

[45] Torkler, S., Wallaschofski, H., Baumeister, S.E., Völzke, H., Dörr, M., Felix, S., Rettig, R., Nauck, M. and Haring, R. (2011) Inverse Association between Total Testosterone Concentrations, Incident Hypertension and Blood Pressure. The Aging Male, 14, 176-182. https://doi.org/10.3109/13685538.2010.529194

[46] Heidelbaugh, J.J. (2016) Endocrinology Update: Testicular Hypogonadism. FP Essentials, 451, 31-41.

[47] Jackson, G., Boon, N., Eardley, I., Kirby, M., Dean, J., Hackett, G., Montorsi, P., Montorsi, F., Vlachopoulos, C., Kloner, R., Sharlip, I. and Miner, M. (2010) Erectile Dysfunction and Coronary Artery Disease Prediction: Evidence-based Guidance and Consensus. International Journal of Clinical Practice, 64, 848-857. 
https://doi.org/10.1111/j.1742-1241.2010.02410.x

[48] Moreno-Villanueva, M. and Bürkle, A. (2014) Molecular Consequences of Psychological Stress in Human Aging. Experimental Gerontology, 68, 39-42.

https://doi.org/10.1016/j.exger.2014.12.003 